\title{
Speech discrimination challenges of healthcare professionals whilst wearing Personal Protective Equipment (PPE) during the coronavirus disease 2019 (COVID-19) pandemic
}

Thomas Hampton ${ }^{1}$, Rosa Crunkhorn ${ }^{1}$, Natalie Lowe ${ }^{1}$, Jaya Bhat ${ }^{1}$, Emma Hogg ${ }^{1}$, Walid Afifi $^{1}$, Madhankumar Krishnan ${ }^{1}$, Ian Street $^{2}$, Sujata DE ${ }^{3}$, Ravi Sharma ${ }^{4}$, Raymond Clarke $^{5}$, Sudhira Ratnayake ${ }^{2}$, Soumit Dasgupta ${ }^{2}$, and Sunil Sharma ${ }^{1}$

${ }^{1}$ Alder Hey Children's Hospital

${ }^{2}$ Alder Hey Children's NHS Foundation Trust

${ }^{3}$ Alder Hey Children's Hospital

${ }^{4}$ Alder Hey Children's Hospital

${ }^{5}$ Alder Hey Children's NHS Foundation trust

May 26, 2020

\begin{abstract}
Background Coronavirus disease 2019(Covid-19) Personal Protective Equipment (PPE) has been reported to effect communication in healthcare settings. We sought to identify those challenges experimentally. Method Bamford-Kowal-Bench speech discrimination in noise performance of healthcare workers was tested under simulated background noise conditions from a variety of hospital environments. Candidates were assessed for speech intelligibility with and without PPE both at normal speech and raised voice. Results There was a significant difference in speech discrimination scores between normal and PPE wearing subjects in theatre simulated background noise levels (70dB). Conclusion PPE wearing can impact communication and efforts should be made to remind staff about this burden and seek alternative communication paradigms, particularly in theatre environments.
\end{abstract}

\section{INTRODUCTION}

During the Coronavirus disease 2019 (Covid-19) pandemic, healthcare professionals have, where supplies exist, delivered care to their patients across the world wearing mandated Personal Protective Equipment $(\mathrm{PPE})^{1}$. The authors anecdotally found communication and understanding wearing PPE to be drastically reduced in clinical areas, and impact on communication of PPE in general has previously been raised in ${\text { popular } \text { press }^{2} \text { and scientific literature }}^{3,4,5}$. We sought to assess these difficulties through a simulated clinical environment model.

In the clinical context, workers frequently speak and communicate with a degree of background noise, unlike the gold standard silent audiological testing booth. Pure tone audiometry (PTA) represents the "gold standard" test for hearing ability and is a good measure of hearing impairment. However, the audiogram generated by the PTA is a poor indicator of speech recognition in noise ${ }^{6}$. PTA measures hearing sensitivity rather than assessing the auditory and hence speech processing ability of the subject, therefore findings do not always correlate with the functional hearing ability of subjects faced with real-world signals and noise, such as speech ${ }^{7}$. A words-in-noise task adds significant cognitive load versus the same task without noise, and as in clinical settings where there is always a degree of background noise, a Speech in Noise (SiN) testing to be a better real world "stress test" of auditory function ${ }^{8}$. 
Rather than a test of hearing, SiN testing for adults can assist clinicians in undertaking assessments of speech understanding in noise. A screening test that uses sentences rather than single words or phonemes is preferred than monosyllabic word lists presented in quiet conditions as the latter have demonstrated limited reliability and lack validity in relation to real-world simulations ${ }^{9,10,11,12,13}$. We sought to identify if there were genuine measurable challenges to speech discrimination wearing Covid-19 PPE in the present study by SiN tests.

\section{METHOD}

We sought to reproduce background noise in the clinical context by adjusting the signal to noise ratio (SNR) during testing. We chose adaptive SNR, using the Bamford-Kowal-Bench (BKB) Sentence Lists read by a clinician $^{14}$ whilst a PARROT machine ${ }^{15}$ produced the background babble noise (simulated speech such as you might hear in a crowded pub or emergency department) at predetermined levels of noise. The PARROT is a portable digital speech screening system used to assess speech discrimination using a range of recognised speech discrimination tests.

The BKB sentences used in this test were published in 1979 as a protocol for testing hearing impaired children and developed as a SiN test by Niquette et al, $2003^{16}$. There are 10 sentences in each list with 18 lists. Each sentence has three or four words which must be repeated by the subject.

A percentage score can be given for how many words are correctly repeated.

To determine background noise levels in our hospital we conducted 2x 30 second sound meter recordings (ATP Digital sound level meter/ 8928, [calibrated by NHS audiometric calibration service, Audiology department, Withington community Hopsital, Manchester]) in 4 discrete environments; The office, the emergency department (ED), the intensive care unit (ITU) and the operating theatre during normal, daylight working hours.

Background noise level minimum and maximums (during daylight hours with regular levels of staff) were recorded as follows:

\section{0-55dB Office 48-66dB ED 50-78dB ITU 53-84dB Theatre}

Five candidates representing our hospital ENT department were chosen, 2 women and 3 men. Age range was 29-49 years. Median age of candidates was 39 years old.

Initial $250 \mathrm{~Hz}-8 \mathrm{kHz}$ Pure tone audiograms were conducted to confirm no significant hearing loss in our 5 candidates, who had no previous otology history or significant co-morbidity.

All testing was conducted in a soundproofed audiometry booth. Baseline Standard BKB sentences were conducted in silence for all candidates without PPE. Scores were $100 \%$ for all candidates.

We then conducted BKB sentence testing whilst each subject wore the facial PPE suitable for aerosol generating procedures (AGPs) (fit-tested FFP3 mask and head visor). All subjects had previously undergone fit testing to ensure that the PPE worn was fitting appropriately for each individual.

The researcher spoke using the BKB word lists whilst wearing AGP PPE. The subject wore the same PPE at a distance of $2 \mathrm{~m}$. The PARROT machine was placed behind and above the head of the researcher (Parrotplus 2 , produced by Soundbyte). Background noise (adult) babble settings were chosen as characteristic values representing different environments as follows:

$45 \mathrm{~dB}$ Office $55 \mathrm{~dB}$ ED $65 \mathrm{~dB}$ ITU $70 \mathrm{~dB}$ Theatre

Each candidate underwent BKB testing at the 4 background noise levels, in three test conditions:

a) Candidate and researcher in normal conditions without PPE, normal researcher voice b) Candidate and researcher both in AGP PPE, normal researcher voice c) Candidate and researcher both in AGP PPE with researcher attempting to raise voice. 
Raised voice was deemed researcher voice raised to the point at which the researcher felt their voice was comprehensible against background noise.

The percentage of key words in the BKB sentences repeated by the candidate was recorded. Each sentence was read once by the researcher and was not repeated.

During day-to-day working and conversation, people do not speak at the same intensity during the whole conversation and background noise also fluctuates rather than remaining at constant levels, hence our decision to use live, fluctuating voice rather than pre-recorded voices amplified to a fixed and constant volume ${ }^{17}$. When speakers adjust their voice to overcome background noise, this is known as the Lombard effect. Although attempting to raise one's voice or shout usually causes only a small increase in volume between $5-10 \mathrm{~dB}^{18}$, we chose to measure the volume of voice produced by the researcher as a secondary outcome measure. This was not our primary concern as attempts to raise one's voice in day to day clinical practice will have both inter and intra-person variability, so we felt the integrity of the simulation was preserved regardless of actual volume levels produced by the researcher.

\section{Statistical analysis}

Primary outcome measures were; 1: differences in BKB sentence results in different stimulated environments; office, ED, ITU and theatre and 2: differences in BKB sentence results with different PPE equipment scenarios; no PPE, wearing PPE and wearing PPE whilst raising voice volume.

Secondary endpoints were; 1: measurement of mean change in voice volume in response to increase in background noise and 2: mean SNRs with different PPE equipment scenarios and environments.

Data were analysed using IBM SPSS Statistics Subscription (IBM, Armonk, USA). Differences in BKB scores for different PPE equipment simulations in each hospital environment were calculated using one-way repeated ANOVA tests. For any environment found to have a statistically significant result, further comparison of differences in BKB sentence results with different PPE equipment scenarios were analysed using the Wilcoxon Signed-Rank test. A p-value of $<0.05$ was considered significant. The Wilcoxon signed-rank test was used as it compares dependant rather than independent samples.

Patient and Public Involvement This research was conducted without patient involvement. Patients were not consulted to develop outcomes or interpret the results as the focus was staff communication. This may occur if this research is expanded to include clinician-patient communication in the future.

\section{RESULTS}

Table 1 presents the BKB results for the 5 people entered into the study.

\section{Table 1}

One-way repeated-measures ANOVA indicated that different levels of PPE use did not significantly alter BKB sentence results in office or ED settings ( $p=0.26$ and $\mathrm{p}=0.58$ respectively) but showed a trend in ITU $(\mathrm{p}=0.06)$. The statistical assumption of sphericity in the ITU setting was marginally violated; an effect perhaps due to the small sample size. If sphericity was assumed, the one-way repeated measured ANOVA test was statistically significant $(\mathrm{F}(2,8)=6.64, \mathrm{p}=0.02, \eta \mathrm{p} 2=0.73)$. Assumption of sphericity was not violated in the theatre setting results $\left(\chi^{2}(2)=3.13, \mathrm{p}=0.21\right)$ and different levels of PPE use significantly altered BKB sentence results $(\mathrm{F}(2,8)=17.16, \mathrm{p}=0.001, \eta \mathrm{p} 2=0.81)$.

A Wilcoxon Signed-Rank test indicated that BKB sentence scores were significantly lower for subjects wearing PPE (median score 58) compared to those without PPE (median score 92) in a theatre simulated environment $(\mathrm{Z}=-2.02, \mathrm{p}=0.04)$. Increasing voice volume whilst wearing PPE significantly increased BKB sentence scores (median score 86) compared to normal speech volume when wearing PPE (median score 58; $\mathrm{Z}=$ $2.03, \mathrm{p}=0.04)$. There was no significant difference in BKB scores when wearing no PPE (median score 92) compared to when raising voice volume whilst wearing PPE (median score 86$)(\mathrm{Z}=-0.68, \mathrm{p}=0.50)$. 
Our secondary outcome measure was mean change in volume of voice when wearing PPE. The increase in background noise rose by $25 \mathrm{db}$ from $45 \mathrm{~dB}$ (simulated office) to $70 \mathrm{~dB}$ (simulated theatre). Our researcher elevated their natural voice by $13-20 \mathrm{~dB}$ without PPE in response to simulated increasing sound levels which correlates with existing studies showing a natural shift to maintain signal-to-noise ratio in human speech ${ }^{18}$.

Mean voice volumes across all simulations tended to increase with PPE wearing and increase again with PPE wearing and raised voice. The mean SNR achieved are shown in Table 2 below:

\section{Table 2}

\section{DISCUSSION}

Our study supports our assumption that wearing facial PPE reduces staff understanding and conventional communication in simulated ITU and theatre settings. Despite the small sample size, results suggest that the louder background environments such as theatre produced the most pronounced (statistically significant) effect on speech comprehension. The excess noise generated in such environments can be attributed to many factors (aptly summarised in the paper by Kam et al, $1994^{19}$ ) that range from equipment and type of surgi$\mathrm{cal} /$ anaesthetic activity to numbers of personnel and consequent raised voice levels. We have demonstrated that wearing PPE will complicate communication further. In this study, speech comprehension wearing PPE within the theatre simulated environment $(70 \mathrm{~dB})$ was significantly worse than wearing no PPE. Raising of voice in a theatre simulated environment when wearing PPE caused a significant improvement of BKB scores to a level that was not significantly different from scores when not wearing PPE.

Despite the observed variance in SNR, BKB scores were still generally poorer with PPE which may indicate a difficulty in understanding un-related to volume or SNR.

Background levels of noise in our simulation were derived from environmental recordings that correlated with prior studies where noise levels in the operating theatre exceed World Health Organisation recommendations ${ }^{20}$. It has been suggested that noise masking speech often results in surgeons having to repeat themselves and consequently, it takes longer for other members of the team to respond or assist ${ }^{20}$. Research exists on background noise and its impact on adherence of staff to the WHO surgical safety checklist, but outside of calls for quiet during the 'Time Out', perceptions of barriers to communication during the rest of the procedure is less well investigated ${ }^{21}$.

We have confirmed anecdotal reports that communication difficulties due to PPE will impact on healthcare workers. Safety of healthcare staff is paramount and the ongoing use of PPE as the initial coivd-19 pandemic wanes is likely to continue. Therefore we anticipate that these communication issues will be exacerbated, particularly in operating theatres, where anaesthetists, operating department practionners, nurses and surgeons will wear AGP PPE for prolonged durations once some longer procedures and elective operating recommences. Interpretation of our findings should be in light of the fact that we simulated background noise and the study candidates were healthcare staff without hearing impairment who regularly work together, which may mean our results would not be reproduced "in the field". Clinical situations regularly involve a variety of shift-working healthcare providers and the additional cognitive load of actually treating patients. Nonetheless, our simulation used validated speech testing and we are unaware of any other studies which have assessed communication difficulties with Covid-19 PPE to this standard.

The importance of speech understanding for achieving success on shared objectives has been extensively researched in military and industrial occupational settings, with need to communicate with co-workers in noisy backgrounds regularly resulting in the removal of protective equipment ${ }^{22}$. There are obvious and immediate implications in theatre e.g. safety compromised, wrong instrument being selected or inadequate delivery of the WHO checklist.

Studies have suggested that as much as $12 \mathrm{~dB}$ SNR is required for speech understanding in the presence of background noise levels up to $110 \mathrm{~dB} \mathrm{SPL}^{23}$ but thresholds for adults have also been recorded with ratios close to $0 \mathrm{~dB}$ or $<0 \mathrm{~dB}^{24}$. 
One impact of PPE we have not discussed is the removal of visual cues to communication. Various studies have demonstrated that visual features strongly affect the perception of $\operatorname{speech}^{17}$. This contribution is most pronounced in noisy environments where the intelligibility of audio-only speech is quickly degraded ${ }^{25}$.

We recommend that regular reminders to speak up and acknowledge communication difficulties at key times during ITU ward rounds and theatre pre-operative briefs may help staff to improve communication whilst wearing PPE. Some specialty guidelines have recommended wearing photographs of staff members over their PPE or writing their name and roles on the apron ${ }^{26}$. Some centres have advocated communicating with hand signals, transparent masks or hoods ${ }^{17}$, white-boards or even two way radio/walkie-talkies in cellophane bags where possible ${ }^{27}$. Others have suggested possible wireless microphone and speaker systems incorporated into $\mathrm{PPE}^{28}$ (micrashell) or even already provided PPE designs with voice amplification solutions ${ }^{29}$ that utilise mobile phone technology. For modern multidisciplinary teams, this may not be a suitable solution when there needs to be multidirectional conversation and information exchange. Solutions like CARDMEDIC (a free to use collection of communication flashcards) have been designed to help healthcare workers speak to patients despite PPE, but we are unaware of any similar device specific to communication between healthcare workers i.e. in theatre (https://www.cardmedic.com/) ${ }^{30}$.

The drawbacks in this study were principally the small sample size that did not allow us to measure an effect size. In addition the study was performed in one hospital site only and representative environmental noise levels could differ across different hospital sites. We welcome the opportunity to work with other teams across the UK and further afield in testing, trialling and simulation, as well as supporting qualitative work for any PPE communication solutions for future working practices.

\section{CONCLUSIONS}

Where attempts to deliberately raise voice or shout through PPE were simulated, understanding significantly improved as expected. Raising of voice for prolonged periods may lead to issues with voice strain and abuse, in addition to frustration or miscommunication. We hope that now communication difficulties have been demonstrated, this will help drive attempts to mitigate these issues for healthcare workers emerging from the Covid-19 pandemic. We hope our findings can inform the ongoing use of PPE once elective healthcare provision is restarted and for the future, facing whatever pandemics may lie ahead.

All authors declare no conflicts of interest, financial or otherwise.

\section{REFERENCES}

1. Public Health England Guidance, COVID-19 personal protective equipment (PPE), 3 May 2020, https://www.gov.uk/government/publications/wuhan-novel-coronavirus-infection-prevention-andcontrol/covid-19-personal-protective-equipment-ppe

2. Cullen, P. Life in an ICU during Covid-19: 'There is no hugging someone who is upset, Irish times, Mar 26, 2020, https://www.irishtimes.com/news/health/life-in-an-icu-during-covid-19-there-is-no-huggingsomeone-who-is-upset-1.4212066

3. Hah, S., Yuditsky, T., Schulz, K. A., Dorsey, H., Deshmukh, A. R., \& Sharra, J. (2009). Evaluation of human performance while wearing respirators (DOT/FAA/TC-09/10). Atlantic City International Airport, NJ: Federal Aviation Administration, William J. Hughes Technical Center. https://hf.tc.faa.gov/publications/2009-evaluation-of-human-performance/full_text.pdf

4. WHO, 2016, Personal protective equipment for use in a filovirus disease outbreak: Rapid advice guideline.World Health Organization. ISBN 9789241549721

5. Ellis, R., Hay-David, A., \& Brennan, P. A. (2020). Operating during the COVID-19 pandemic: How to reduce medical error. The British journal of oral \& maxillofacial surgery, S0266-4356(20)30146-7. Advance online publication. https://doi.org/10.1016/j.bjoms.2020.04.002

6. Vermiglio, A., Soli, S., Freed, D. \& Fisher, L. (2012) The relationship between high-frequency puretone hearing loss, hearing in noise test (HINT) thresholds, and the articulation index. Journal of the American Academy of Audiology. 768 23:10:779-88 
7. Musiek, F., Shinn, J., Chermak, G. \& Bamiou, D-E. (2017) Perspectives on the Pure-Tone Audiogram. Journal of the American Academy of Audiology, DOI: https://doi.org/10.3766/jaaa.16061

8. Wilson, R. (2011) Clinical Experience with the Words-in-Noise Test on 3430 Veterans: Comparisons with Pure-Tone Thresholds and Word Recognition in Quiet. Journal of the American Academy of Audiology. 22:405-423

9. Walden, B., Schwartz, D., Williams, D., et al (1983). Test of the assumption underlying comparative hearing aid evaluations. Journal of Speech and Hearing Disorders. 48:264-273

10. Nilsson, M., Soli, S. \& Sullivan, J. (1994) Development of the Hearing in Noise Test for the measurement of speech reception thresholds in quiet and in noise. Journal of the Acoustical Society of America. 95(2), 1085-1099

11. Beattie, R., Barr, T., \& Roup, C. (1997). Normal and hearing impaired word recognition scores for monosyllabic words in quiet and noise. British Journal of Audiology. 31:153-164

12. Taylor, B. (2003) Speech-in-noise tests: How and why to include them in your basic test battery. The Hearing Journal. 56:1:40-43

13. Killion M., Niquette P., Gudmundsen G., Revit L. \& Banerjee S. (2004) Development of a quick speechin-noise test for measuring signal-to-noise ratio loss in normal-hearing and hearing-impaired listeners. Journal of the Acoustical Society of America. 116(4):2395-2405

14. Bench, J., Kowal, A. \& Bamford, J. (1979). The BKB (Bamford-Kowal-Bench) Sentence Lists for partially-hearing children. British Journal of Audiology. 13, 108-112

15. Shaw P. The Parrot Speech Discrimination Test. Br Soc Audiol News 1997. 20: 44-45

16. Niquette, P., Arcaroli, J., Revit, L., Parkinson, A., Staller, S., Skinner, M. \& Killion, M. (2003) Development of the BKB-SIN Test. Paper presented at the annual meeting of the American Auditory Society, Scottsdale, AZ

17. Atcherson, S. R., Mendel, L. L., Baltimore, W. J., Patro, C., Lee, S., Pousson, M., \& Spann, M. J. (2017). The Effect of Conventional and Transparent Surgical Masks on Speech Understanding in Individuals with and without Hearing Loss. Journal of the American Academy of Audiology, 28(1), 58-67. doi:10.3766/jaaa.15151

18. Bottalico, P., Passione, I. I., Graetzer, S., \& Hunter, E. J. (2017). Evaluation of the starting point of the Lombard Effect. Acta acustica united with acustica: the journal of the European Acoustics Association (EEIG), 103(1), 169-172. https://doi.org/10.3813/AAA.919043

19. Kam PCA., Kam AC., Thompson JF. Noise pollution in the anaesthetic and intensive care environment. Anaesthesia, 1994, Volume 49, pages 982-98

20. Weldon, Sharon-Marie \& Korkiakangas, Terhi \& Bezemer, Jeff. (2015). Music and communication in the operating theatre. Journal of Advanced Nursing. 71. 10.1111/jan.12744.

21. Schwendimann R, Blatter C, Lüthy M, et al. Adherence to the WHO surgical safety checklist: an observational study in a Swiss academic center. Patient Saf Surg. 2019;13:14. Published 2019 Mar 12. doi:10.1186/s13037-019-0194-4

22. Le Prell, C \& Clavier O, Effects of noise on speech recognition: Challenges for communication by service members, Hearing Research, 349 (2017) 76-89 https://doi.org/10.1016/j.heares.2016.10.004

23. Robinson, G and Casali, J, Speech communications and signal detection in noise In: E.H. Berger, L.H. Royster, J.D. Royster, D.P. Driscoll, M. Layne (Eds.), The Noise Manual (fifth ed.), American Industrial Hygiene Association, Fairfax (2003), pp. 567-600

24. Neuman, Arlene C; Wroblewski, Marcin; Hajicek, Joshua; Rubinstein, Adrienne, Combined Effects of Noise and Reverberation on Speech Recognition Performance of Normal-Hearing Children and Adults, Ear and Hearing: June 2010, Vol31,3, p336-344 doi: 10.1097/AUD.0b013e3181d3d514

25. M. McClain, K. Brady, M. Brandstein and T. Quatieri, "Automated lip-reading for improved speech intelligibility," 2004 IEEE International Conference on Acoustics, Speech, and Signal Processing, Montreal, Que., 2004, pp. I-701.

26. RCSLT guidance on personal protective equipment (PPE) and COVID-19, 1 May $2020 \quad$ https://www.rcslt.org/-/media/docs/Covid/RCSLT-PPE-guidance-1-May2020.pdf?la=en\&hash=76CF9CA7A4BB991FE60CEAD35B3940895E8472F6 
27. Liew, M.F., Siow, W.T., MacLaren, G. et al. Preparing for COVID-19: early experience from an intensive care unit in Singapore. Crit Care 24, 83 (2020). https://doi.org/10.1186/s13054-020-2814-x 28. https://production.club/micrashell/

29. https://www.pneumask.org/clinician-engagement\#voice-amplification

30. https://www.cardmedic.com/

\section{Hosted file}

Table 1.docx available at https://authorea.com/users/326310/articles/454193-speechdiscrimination-challenges-of-healthcare-professionals-whilst-wearing-personal-

protective-equipment-ppe-during-the-coronavirus-disease-2019-covid-19-pandemic

\section{Hosted file}

Table 2.docx available at https://authorea.com/users/326310/articles/454193-speechdiscrimination-challenges-of-healthcare-professionals-whilst-wearing-personalprotective-equipment-ppe-during-the-coronavirus-disease-2019-covid-19-pandemic 